\title{
Growth models, random matrices and Painlevé transcendents
}

\author{
Peter J. Forrester*1 \\ *Department of Mathematics and Statistics, University of Melbourne, \\ Victoria 3010, Australia
}

The Hammersley process relates to the statistical properties of the maximum length of all up/right paths connecting random points of a given density in the unit square from $(0,0)$ to $(1,1)$. This process can also be interpreted in terms of the height of the polynuclear growth model, or the length of the longest increasing subsequence in a random permutation. The cumulative distribution of the longest path length can be written in terms of an average over the unitary group. Versions of the Hammersley process in which the points are constrained to have certain symmetries of the square allow similar formulas. The derivation of these formulas is reviewed. Generalizing the original model to have point sources along two boundaries of the square, and appropriately scaling the parameters gives a model in the KPZ universality class. Following works of Baik and Rains, and Prähofer and Spohn, we review the calculation of the scaled cumulative distribution, in which a particular Painlevé II transcendent plays a prominent role.

\section{Introduction}

The aim of this review is to explain aspects of developments over the past few years relating some observables in statistical mechanics models to random matrix averages and then to Painlevé transcendents. In addition to the theoretical interest in these inter-relationships, the fact that the Painlevé transcendents are readily computable means that quantitative predictions for certain order one scaled observables are available for the first time. Perhaps the most significant such result is the calculation due to Prähofer and Spohn [33] of the exact two-point scaling function for one-dimensional stationary KPZ (Kardar-Parisi-Zhang) growth. KPZ growth is generally believed (see e.g. [25, 9]) to underlie a diverse number of growth models in $1+1$ dimension. One model within the KPZ universality is the polynuclear growth (PNG) model. The particular variant of the latter relevant in this context was solved in terms of a Painlevé transcendent known from random matrix theory [37] by Baik and Rains [7, and it is this solution which is interpreted and computed in 33. Section 4 of the present work gives some details of the exact solution.

We begin in Section 2 by reviewing the calculation of the cumulative distribution for the longest path in the Hammersley process. This will be shown to be equivalent to computing the cumulative distribution for the maximum height in the PNG model, the longest increasing subsequence length for a random permutation, or the maximum displacement of certain families

\footnotetext{
${ }^{1}$ Supported by the Australian Research Council
} 
of non-intersecting paths. The cumulative distribution is given as a particular random matrix average over the unitary group. In Section 3, four different symmetrizations of the Hammersley process are considered. The cumulative distributions of the longest path in each of these cases can again be written as particular random matrix averages, involving the orthogonal and symplectic groups in two of the cases, and the unitary group in the remaining two. After presenting some details of the calculation of the scaled distribution for the variant of the PNG model of relevance to KPZ growth in Section 4, we conclude in Section 5 by indicating aspects of the Painlevé transcendent content of the averages over the orthogonal and symplectic groups encountered in Section 2 .

\section{The Hammersley process}

\subsection{Relationship to permutations}

The Hammersley process (see [2, 3] for an extended account of different emphasis to that given here, and for references to the original literature) refers to the following stochastic model. In the unit square mark in points uniformly at random according to a Poisson rate with intensity $\lambda^{2}$, so that the probability the square contains $N$ points is equal to $\lambda^{2 N} e^{-\lambda^{2}} / N$ !. Form a continuous path by joining points with straight line segments of positive slope, which are thus orientated up and to the right. Extend this path to begin at $(0,0)$ and finish at $(1,1)$ by adding an extra segment at both ends, and define the length of the extended path as the number of points it contains. Take as the primary observable quantity the stochastic variable, $l^{\square}$ say, specifying the maximum of the lengths of all possible extended paths (see Figure 1).

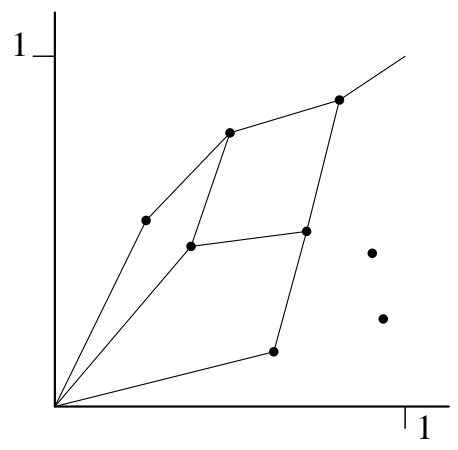

Figure 1: Eight points in the unit square, and the extended directed paths of maximum length. Since the number of points in these paths equals three, here $l_{n}^{\square}=3$.

For any particular realization of exactly $N$ points the Hammersley process gives a geometrical construction of a random permutation of $\{1,2, \ldots, N\}$. This comes about by first labelling the $x$ coordinates of the points by $0<x_{1}<\cdots<x_{N}<1$ and similarly the $y$ coordinates by $0<y_{1}<\cdots<y_{N}<1$. Each point will then have a coordinate of the form $\left(x_{j}, y_{P(j)}\right)$ where $\{P(1), \ldots, P(N)\}$ is a permutation of $\{1,2, \ldots, N\}$. The quantity $l^{\square}$ also has an interpretation in terms of the permutation. Thus the analogue of an up/right path connecting points is a 
subsequence $1 \leq j_{1}<j_{2}<\cdots<j_{r} \leq N$ such that $P\left(j_{1}\right)<P\left(j_{2}\right)<\cdots<P\left(j_{r}\right)$, which is referred to as an increasing subsequence. The length of an increasing subsequence is defined as the value $r$. We then see from the definitions that the maximum length of all increasing subsequences of $P$ coincides with $l^{\square}$.

\subsection{Polynuclear growth model}

Consider the $x$ - $t$ half plane $t>0$. Let this half plane be filled with points uniformly at random and such that the mean density is unity. These points are to be thought of as seeds for nucleation events of layered growth. In a droplet model, at $(x, t)=(0,0)$ a single layer, taken to have zero height, starts spreading with unit velocity to the left and to the right. Forming on top of the ground layer are new layers of unit height. These layers, or parts thereof, are formed at spacetime positions $\left(x_{i}, t_{i}\right)$ for each nucleation event bounded by the 'lightcone' axis $u=(t+x) / \sqrt{2}$, $v=(t-x) / \sqrt{2}$; nucleation events outside this cone are not created at a time that their position coordinate makes contact with the ground layer or its growth. The nucleation events $\left(x_{i}, t_{i}\right)$ inside the lightcone create the beginning of a portion of a layer of unit height on top of the ground layer, or existing layers, at position $x_{i}$. The layers are formed by the growth of the nucleation events with unit velocity to the left and to the right; if two growing portions of a layer collide, then growth at that point ceases and the two portions become one, growing only at the end points of this one portion (see Figure 2 for an example). Of interest is the statistical properties of the height at the origin after this growth process - known as the polynuclear growth (PNG) model - has been underway for time $t=T$.
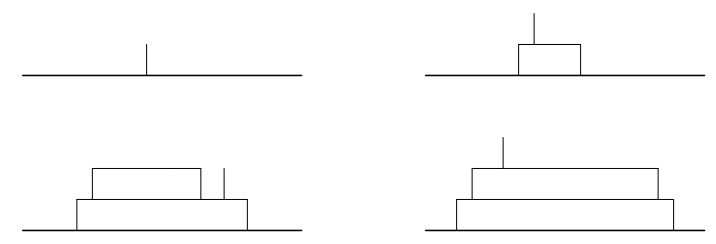

Figure 2: Example of the plateau profile at the time of four successive nucleation events, including the initial event (which is labelled the 0th event and its plateau the 0th level). Note that between the second and third nucleation event, two plateaus on the first level have coalesced.

The first observation is that only those nucleation events in the region $[u=0, u=T / \sqrt{2}] \times$ $[v=0, v=T / \sqrt{2}]$ of the lightcone can contribute to the height at $x=0$ up to time $t=T$. Suppose in a realization of the nucleation events there are $N$ points in this region. For a Poisson process of unit density this occurs with probability $\lambda^{2 N} e^{-\lambda^{2}} / N$ !, where $\lambda^{2}=T^{2} / 2$ is the area of the region. Use the construction of the previous subsection to associate with the configuration of points a permutation $P$ (see Figure 3). Also indicated in Figure 3 are the world lines of the nucleation events, which show clearly the layered structure of the growth, and in particular the height at the origin after time $T$. The layers in which the particular nucleation events occur are simply related to the permutation $P$. This is done by partitioning the permutation 
into decreasing subsequences using the leftmost digits at all times. The $j$ th such decreasing subsequence corresponds to the $j$ th layer in the growth process. For example, in Figure 3 the permutation is 5374162, and the decreasing subsequences formed from the leftmost digits are (531)(742)(6). It is easy to see that in general the number of decreasing subsequences of this type is equal to the length of the longest increasing subsequence of the same permutation. Thus studying the height at the origin in the PNG model after time $T$ is equivalent to studying the maximum path length in the Hammersley process with intensity $\lambda^{2}=T^{2} / 2$.

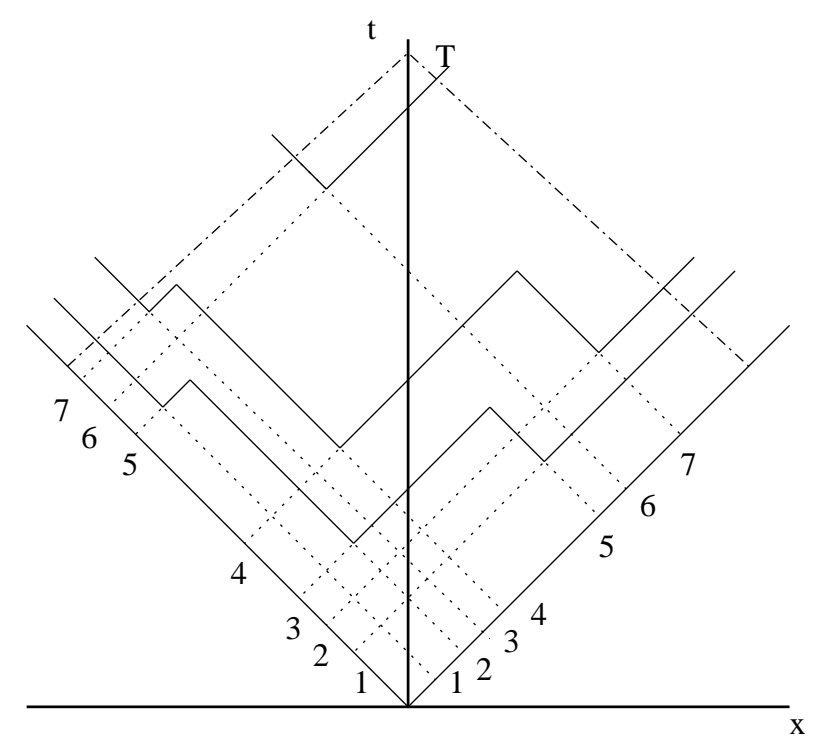

Figure 3: World lines for the endpoints of the plateaux. Only nucleation events inside the square shaped region including the lines $x= \pm t$, and the lines from $t=T$ to these lines affect the height at the origin. The nucleation points occur at $\mathrm{v}$ shaped configurations, while the inverted $\mathrm{v}$ part of the worldlines correspond to the joining of the plateaux originating from two different nucleation events. The labelling on the lines $x=t$ and $x=-t$ allow the world lines to be identified uniquely with a permutation.

\subsection{Robinson-Schensted-Knuth correspondence}

To analyze the Hammersley process requires a formula for the number of up/right paths less than some prescribed value $l$ say. For this purpose, in a realization containing $N$ points, we first associate with each point $\left(x_{j}, y_{P(j)}\right), j=1, \ldots, N$ the permutation matrix defined so that the entries $(j, P(j))$ are equal to unity with all other entries equal to zero. We then apply what is essentially Viennot's shadow method [0] to give a bijection between permutation matrices and certain configurations of lattice paths, the outermost member of which can be interpreted as the profile of a lattice variant of the PNG model [30].

In fact it is possible to give a bijection between a $n \times n$ non-negative integer matrix $X=$ $\left[x_{i, j}\right]_{i, j=1, \ldots, n}$ and a pair of so called up/ right horizontal $(\mathrm{u} / \mathrm{rh})$ non-intersecting lattice paths. 
This bijection is equivalent to the celebrated Robinson-Schensted-Knuth correspondence mapping $X$ to a pair of semi-standard tableaux [22]. The non-intersecting lattice paths are defined on the square lattice and start at $x=0$, one unit apart in the $y$-direction at $y=0, \ldots,-(n-1)$, and finish at $x=n-1$, with $y$-coordinates $\mu_{l}-(l-1)(l=1, \ldots, n)$ where $\mu_{1} \geq \mu_{2} \geq \cdots \geq \mu_{N} \geq 0$. The path starting at $y=-(l-1)$ is referred to as the level- $l$ path. Each path may move either up or to the right along the edges of the lattice, with the constraint that the paths may not intersect. When $X$ is a permutation matrix and so has exactly one non-zero entry, equal to unity, in each row and column the paths are further restricted so that for each allowed $x$-value there is exactly one up step in total, which is of unit length.

In the general case the entries $x_{i, j}$ of $X$, where for convenience the rows are labelled from the bottom, represent the heights of columns of unit length centred about $x=j-i$ which occur at time $t=i+j-1$. This labelling is simple to implement by first rotating the matrix $45^{\circ}$ anticlockwise. The columns are to be placed on top of the level-1 path formed by earlier nucleation events and their growth. On this latter point, during each time interval the existing profile or profiles are required to grow one unit to the left or to the right, with any resulting overlap recorded on the path one level below. What results are a set of at most $n$ non-intersecting lattice paths, equivalent to a pair of $\mathrm{u} / \mathrm{rh}$ non-intersecting lattice paths each with the same final positions. Furthermore, it is easy to see that the process is invertible, and so there is a bijection between non-negative integer matrices and pairs of $\mathrm{u} / \mathrm{rh}$ non-intersecting lattice paths. As already remarked, in the case of permutation matrices, these paths have the additional constraint of containing exactly one up step in total for each allowed $x$-value, $x= \pm(2 n+3 / 2-2 j)$ $(j=1, \ldots, n)$. An example is given in Figure 4.

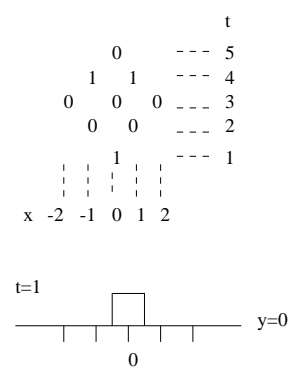

$t=3$

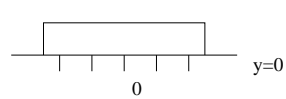

$\mathrm{t}=4$

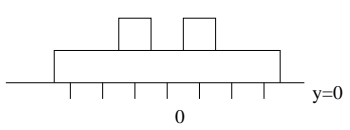

$\mathrm{t}=2$

$\mathrm{t}=5$

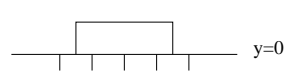

te

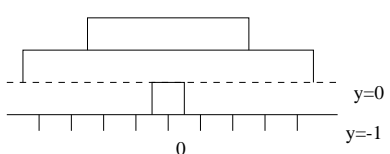

Figure 4: The RSK mapping in the non-intersecting paths picture from a permutation matrix to a pair of $\mathrm{u} / \mathrm{rh}$ non-intersecting paths, constrained so that there is exactly one up set in total for each allowed value of $x$.

Non-intersecting $\mathrm{u} / \mathrm{rh}$ lattice paths, constrained to have exactly one up step in total for each allowed $x$-value can be encoded as standard tableaux. The latter consist of an array of unit boxes stacked across rows of length $\mu_{1}, \mu_{2}, \ldots, \mu_{n}$ respectively with the first box in each row 
contained in the first column of the array etc. The standard tableau is said to have shape $\mu$. One plus the value of the $x$-coordinates that contain up steps are marked in order along row $l$. Thus the numbers in the array have the property of being strictly increasing both along rows and down columns, with each number from $\{1,2, \ldots, n\}$ recorded once. The standard tableau is said to have content $n$.

Let $f_{N}^{\mu}$ denote the number of standard tableaux of shape $\mu$ and content $N$. Let $l_{N}^{\square}$ denote the longest increasing subsequence length in a realization of the Hammersley process containing $N$ points. Then it follows from the correspondence with pairs of constrained $\mathrm{u} / \mathrm{rh}$ lattice paths and thus pairs of standard tableau that

$$
\operatorname{Pr}\left(l_{N}^{\square} \leq l\right)=\frac{1}{N !} \sum_{\mu: \mu_{1} \leq l}\left(f_{N}^{\mu}\right)^{2} .
$$

As a first step towards evaluating the sum in (2.1), note that the symmetry between rows and columns in a standard tableau implies we can write

$$
\operatorname{Pr}\left(l_{N}^{\square} \leq l\right)=\frac{1}{N !} \sum_{\mu: \mu_{1}^{\prime} \leq l}\left(f_{N}^{\mu}\right)^{2}
$$

where $\mu_{1}^{\prime}$ denotes the length of the first column. In terms of lattice paths, this can be understood from the bijection between $\mathrm{u} / \mathrm{rh}$ lattice paths and dual $\mathrm{u} / \mathrm{rh}$ lattice paths, where if in the former the maximum displacement is $l$, then in the latter there are exactly $l$ lattice paths (see Figure 51).

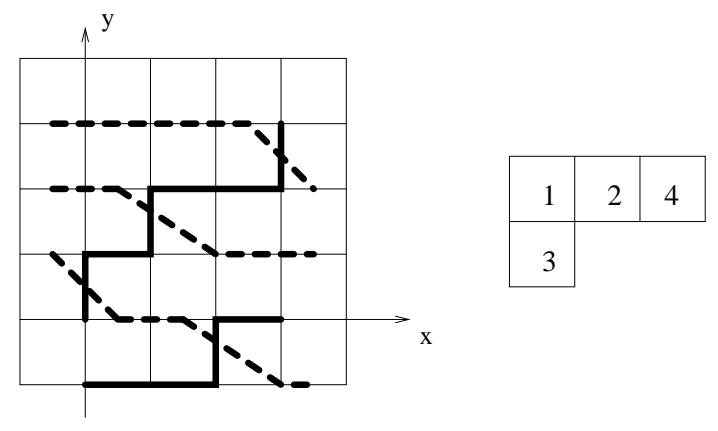

Figure 5: Drawn in heavy lines on the square lattice is a family of two $\mathrm{u} / \mathrm{rh}$ lattice paths, constrained so that there is exactly one up step in total for each $x$-value, and with maximum displacement 3 units, while drawn in heavy dashed lines are the corresponding three dual lattice paths. Also given is the standard tableau encoding the two original $\mathrm{u} / \mathrm{rh}$ lattice paths.

\subsection{Relationship to the lock step model of vicious walkers}

The task of computing the number of pairs of constrained $\mathrm{u} / \mathrm{rh}$ lattice paths, each with the same final positions, and containing exactly $l$ lattice paths can be considered as a special case of a more general counting problem. First we remark that a set of $l^{*}<l$ lattice paths between $x=0$ to $x=n-1$ can be uniquely converted to a set of $l$ lattice paths by drawing horizontal 
segments from $x=0$ to $x=n-1$ along $y=-\left(l^{*}-1\right), \ldots,-(l-1)$, so to compute (2.2) the case of precisely $l$ lattice paths is what is relevant. We generalize the rules of constructing the lattice paths so that for each allowed $x$ value $x=0,1, \ldots, n-1$ there is again only one segment which is not horizontal, but now this segment may be either up or down, subject again to the non-intersecting constraint. These paths can be considered as the world lines for the stochastic evolution of $l$ random walkers on a one-dimensional lattice. At each tick of the clock exactly one walker may move to the left or to the right one lattice space, subject to the constraint that this lattice site is not already occupied. This defines what is known as the random turns model of vicious walkers [12, 13]. Let an up (down) step at $x=j-1$ be weighted $w_{j}^{+}\left(w_{j}^{-}\right)$, and suppose furthermore that each level- $k$ path returns to $y=-(k-1)$ at $x=n-1$. Then with

$$
\left.G_{l, n}:=\sum_{\text {paths }} \prod_{k=1}^{l} \text { (weight of level- } k \text { path }\right)
$$

it is shown in [13, 15] that

$$
G_{l, n}=\frac{1}{(2 \pi)^{l} l !} \int_{-\pi}^{\pi} d \theta_{1} \cdots \int_{-\pi}^{\pi} d \theta_{l} \prod_{k=1}^{n} \sum_{j=1}^{l}\left(w_{k}^{+} e^{i \theta_{j}}+w_{k}^{-} e^{-i \theta_{j}}\right) \prod_{1 \leq j<k \leq l}\left|e^{i \theta_{k}}-e^{i \theta_{j}}\right|^{2} .
$$

But

$$
\sum_{\mu: \mu_{1}^{\prime} \leq l}\left(f_{N}^{\mu}\right)^{2}=G_{l, 2 N} \mid \begin{gathered}
w_{p}^{-}=0, w_{p}^{+}=1(p=1, \ldots, N) \\
w_{p}^{+}=0, w_{p}^{-}=1(p=N+1, \ldots, 2 N)
\end{gathered}
$$

so we see from (2.2) and (2.3) that

$$
\begin{aligned}
\operatorname{Pr}\left(l_{N}^{\square} \leq l\right) & =\frac{1}{N !} \frac{1}{(2 \pi)^{l} l !} \int_{-\pi}^{\pi} d \theta_{1} \cdots \int_{-\pi}^{\pi} d \theta_{l}\left|\sum_{j=1}^{l} \exp i \theta_{j}\right|^{2 N} \prod_{1 \leq j<k \leq l}\left|e^{i \theta_{k}}-e^{i \theta_{j}}\right|^{2} \\
& =\frac{N !}{(2 N) !}\left\langle\left(\sum_{j=1}^{l} 2 \cos \theta_{j}\right)^{2 N}\right\rangle_{U(l)}
\end{aligned}
$$

where $\langle\cdot\rangle_{U(l)}$ denotes an average over the eigenvalue probability density function for random matrices from the classical group $U(l)$,

$$
\frac{1}{(2 \pi)^{l} l !} \prod_{1 \leq j<k \leq l}\left|e^{i \theta_{k}}-e^{i \theta_{j}}\right|^{2}, \quad-\pi<\theta_{j}<\pi .
$$

To see the validity of the second equality in (2.4), one first notes

$$
\left(\sum_{j=1}^{l} 2 \cos \theta_{j}\right)^{2 N}=\sum_{p=0}^{2 N}\left(\begin{array}{c}
2 N \\
p
\end{array}\right)\left(\sum_{j=1}^{l} e^{i \theta_{j}}\right)^{p}\left(\sum_{j=1}^{l} e^{-i \theta_{j}}\right)^{2 N-p}
$$

and then observes that only the $p=N$ term in this sum is non-zero after averaging over $U(l)$. Now, according to the definitions

$$
\operatorname{Pr}\left(l^{\square} \leq l\right)=e^{-\lambda^{2}} \sum_{N=0} \frac{\lambda^{2 N}}{N !} \operatorname{Pr}\left(l_{N}^{\square} \leq l\right)
$$

so substituting (2.4) gives 23, 34

$$
\operatorname{Pr}\left(l^{\square} \leq l\right)=e^{-\lambda^{2}}\left\langle e^{\lambda \operatorname{Tr}\left(U+U^{\dagger}\right)}\right\rangle_{U \in U(l)} .
$$




\subsection{Relationship to Schur polynomials}

Consider the set of all semi-standard tableaux of shape $\lambda$ and content $N$, with each occurence of $j$ in the numbering therein weighted $w_{j}$. The total weight

$$
s_{\lambda}\left(w_{1}, \ldots, w_{N}\right):=\sum_{\substack{\text { semi-standard tableux } \\ \text { shape } \lambda \text {,content } N}} w_{1}^{\# 1^{\prime} s} w_{2}^{\# 2^{\prime} s} \cdots w_{N}^{\# N^{\prime} s}
$$

is a symmetric polynomial in $w_{1}, \ldots, w_{N}$ known as the Schur polynomial. Because in a standard tableaux each number occurs exactly once, it follows immediately that

$$
\left[w_{1} w_{2} \cdots w_{N}\right] s_{\lambda}\left(w_{1}, \ldots, w_{N}\right)=f_{N}^{\lambda}
$$

where $\left[w_{1} w_{2} \cdots w_{N}\right]$ denotes the coefficient of $w_{1} w_{2} \cdots w_{N}$. This fact allows expressions such as (2.1) involving $f_{N}^{\mu}$ to be evaluated as special cases of Schur function identities. Explicitly, in relation to (2.1) use can be made of the Schur function identity

$$
\sum_{\mu: \mu_{1} \leq l} s_{\mu}\left(a_{1}, \ldots, a_{N}\right) s_{\mu}\left(b_{1}, \ldots, b_{N}\right)=\left\langle\prod_{j=1}^{N} \prod_{k=1}^{l}\left(1+a_{j} e^{i \theta_{k}}\right)\left(1+b_{j} e^{-i \theta_{k}}\right)\right\rangle_{U(l)} .
$$

As to be revised in Section 4, this has direct relevance to the Johansson model. Our present interest is that by extracting the coefficient of $a_{1} \cdots a_{N} b_{1} \cdots b_{N}$ from both sides, making use of (2.7) on the left hand side, it follows that

$$
\sum_{\mu: \mu_{1} \leq l}\left(f_{N}^{\mu}\right)^{2}=\left\langle\left|\sum_{k=1}^{l} e^{i \theta_{k}}\right|^{2 N}\right\rangle_{U(l)}
$$

in accordance with the evaluation of (2.1) implied by the first equality in (2.4).

\subsection{A relationship to eigenvalue distributions}

It is of interest to note that the random matrix average over $U(l)$ in (2.5) also arises in another probabilistic setting [14. Thus consider the Laguerre unitary ensemble, specified by the eigenvalue probability density function proportional to

$$
\prod_{l=1}^{N} \lambda_{l}^{a} e^{-\lambda_{l}} \prod_{1 \leq j<k \leq N}\left(\lambda_{k}-\lambda_{j}\right)^{2}, \quad \lambda_{l}>0
$$

For $a=n_{1}-n_{2}, n_{1} \geq n_{2}$, it is realized by the eigenvalues of the random matrix $X^{\dagger} X$, where $X$ is an $n_{1} \times n_{2}$ complex Gaussian matrix. Let $E_{2}^{\mathrm{L}}(s ; a ; N)$ denote the probability that there are no eigenvalues in the interval $(0, s)$. Then it was shown in [14] that the scaled probability

$$
E_{2}^{\mathrm{hard}}(\lambda ; a):=\lim _{N \rightarrow \infty} E_{2}^{\mathrm{L}}\left(\frac{\lambda}{N} ; a ; N\right)
$$


is given by the right hand side of (2.5) with $l=a$ and $\lambda \mapsto \sqrt{\lambda}$ (the superscript "hard" is used to denote the fact the eigenvalue density is strictly zero to the left of $\lambda=0$ ). This fact has been used in [1] to give a straightforward proof that

$$
\lim _{\lambda \rightarrow \infty} \operatorname{Pr}\left(\frac{l^{\square}-2 \lambda}{\lambda^{1 / 3}} \leq s\right)=F_{\mathrm{GUE}}(s) .
$$

where $F_{\mathrm{GUE}}(s)$ is the scaled cumulative distribution of the largest eigenvalue for large random Hermitian matrices with complex Gaussian matrices, a celebrated result due originally to Baik, Deift and Johansson [5], which gives as a corollary the scaled distribution of the longest increasing subsequence of a random permutation.

\section{Symmetrizations of the Hammersley process}

\subsection{Four symmetries of the square}

Baik and Rains [7] have formulated and analyzed four symmetrized versions of the Hammersley process, in which the points are constrained to have particular reflection symmetries of the square. In these symmetrizations, the points are constrained to be invariant under reflections about the diagonal $(0,0)$ to $(1,1)$; diagonal $(0,1)$ to $(1,0)$; both these diagonals; and about the centre point $(1 / 2,1 / 2)$. The four cases are denoted $\square, \nabla, \square$ and $₫$ respectively. The first three of these cases can be further generalized to allow independent Poisson rates for points forming on the diagonal(s). Let us consider each case separately.

\subsection{The symmetry $\square$}

First we specify the Poisson process by which the points are added to the diagonal and below the diagonal. We start with a time interval $[0, z]$, which is broken up into $M$ smaller intervals of equal size, the latter being labelled $j=1, \ldots, M$. In each of these smaller intervals, add one point below the diagonal (together with its image above the diagonal) with probability $z^{2}(j-1 / 2) / M^{2}$ and a point on the diagonal with probability $\alpha z / M$. The probability that there are $n$ points in the square is then given by the coefficient of $w^{n}$ in

$$
\prod_{j=1}^{M}\left(1-\frac{\alpha}{M}+\frac{\alpha w}{M}\right)\left(1-\frac{(j-1 / 2) z^{2}}{M^{2}}+\frac{(j-1 / 2) z^{2} w^{2}}{M^{2}}\right) .
$$

It follows by taking the limit $M \rightarrow \infty$ in this expression that the probability of their being exactly $n$ points after time $z$, when the points below the diagonal are added with rate $z d z$ and those below the diagonal with rate $\alpha d z$ is given by

$$
e^{-\alpha z} e^{-z^{2} / 2} \frac{z^{n}}{n !} \sum_{m=0}^{[n / 2]} \alpha^{n-2 m} s_{n, m}, \quad s_{n, m}:=\left(\begin{array}{c}
n \\
2 m
\end{array}\right) \frac{(2 m) !}{2^{m} m !} .
$$

Furthermore, one sees that this probability conditioned so that there are $n-2 m$ points on the diagonal is equal to the $m$ th term in this sum. 
Let $l_{n, m}^{\square}$ denote the longest path length in a realization of the $\square$ symmetrized Hammersley process consisting of a total of $n$ points, $m$ of which are below the diagonal. It follows from (3.1) and the sentence below that if the points are chosen according to the Poisson process specified above, then after time $z$

$$
\operatorname{Pr}\left(l^{\square} \leq l\right)=e^{-\alpha z-z^{2} / 2} \sum_{n=0}^{\infty} \frac{z^{n}}{n !} \sum_{m=0}^{[n / 2]} \alpha^{n-2 m} s_{n, m} \operatorname{Pr}\left(l_{n, m}^{\square} \leq l\right) .
$$

To compute $\operatorname{Pr}\left(l_{n, m}^{\square} \leq l\right)$ we first note that the sought realizations of the $\square$ symmetrized Hammersley process are in correspondence with $n \times n$ permutation matrices constrained to have $n-2 m$ non-zero entries on the diagonal, and which are furthermore symmetric about the diagonal (recall that our convention is to count rows from the bottom and so the diagonal runs from the bottom left to the top right). It is straightforward to show that there are $s_{n, m}$ distinct such permutation matrices. Next we make a correspondence between such permutation matrices and pairs of suitably constrained $\mathrm{u} / \mathrm{rh}$ lattice paths, or equivalently pairs of suitably constrained standard tableaux.

Now it is immediate from the rules of the PNG model that if the non-negative integer matrix $X=\left[x_{i, j}\right]_{i, j=1, \ldots, n}$ maps to a pair of $\mathrm{u} / \mathrm{rh}$ paths $\left(P_{1}, P_{2}\right)$, then the transposed matrix $X^{T}=\left[x_{j, i}\right]_{i, j=1, \ldots, n}$ maps to a pair of $\mathrm{u} / \mathrm{rh}$ paths $\left(P_{2}, P_{1}\right)$. Thus in the case that $X=X^{T}$ one has $P_{1}=P_{2}$ so the mapping then is to a single family of paths. (The example of Figure 4 exhibits this.) Furthermore, for symmetric non-negative integer matrices $X$ it is a known property of the RSK correspondence that 31

$$
\sum_{j=1}^{n} x_{j, j}=\sum_{j=1}^{n}(-1)^{j-1} \mu_{j}
$$

a fact which can also be derived within the setting of the PNG model [18. Hence it follows that

$$
\begin{aligned}
\operatorname{Pr}\left(l_{n, m}^{\square} \leq l\right) & =\frac{1}{s_{n, m}}\left[\alpha^{n-2 m}\right] \sum_{\mu: \mu_{1} \leq l} \alpha^{\sum_{j=1}^{n}(-1)^{j-1} \mu_{j}} f_{n}^{\mu} \\
& =\frac{1}{s_{n, m}}\left[\alpha^{n-2 m} q_{1} \cdots q_{n}\right] \sum_{\mu: \mu_{1} \leq l} \alpha^{\sum_{j=1}^{n}(-1)^{j-1} \mu_{j}} s_{\mu}\left(q_{1}, \ldots, q_{n}\right)
\end{aligned}
$$

where the second equality follows from (2.7). Now we know from [7] that

$$
\sum_{\mu: \mu_{1} \leq l} \alpha^{\sum_{j=1}^{n}(-1)^{j-1} \mu_{j}} s_{\mu}\left(q_{1}, \ldots, q_{n}\right)=\left\langle\prod_{k=1}^{l}\left(\left(1+\alpha e^{i \theta_{k}}\right) \prod_{j=1}^{n}\left(1+q_{j} e^{i \theta_{k}}\right)\right)\right\rangle_{\mathrm{O}(l)} .
$$

In (3.5) the average over the classical group $\mathrm{O}(l)$ breaks into two parts,

$$
\langle\cdot\rangle_{\mathrm{O}(l)}=\frac{1}{2}\left(\langle\cdot\rangle_{\mathrm{O}^{+}(l)}+\langle\cdot\rangle_{\mathrm{O}^{-}(l)}\right)
$$

where $\langle\cdot\rangle_{\mathrm{O}^{+}(l)}$ denotes an average with respect to the eigenvalue p.d.f. for random matrices from 
the classical group $\mathrm{O}^{+}(l)$,

$$
\begin{aligned}
& \frac{1}{\pi^{l / 2} 2^{l-1}(l / 2) !} \prod_{j=1}^{l / 2} \delta\left(\theta_{j}-\theta_{l / 2+j}\right) \prod_{1 \leq j<k \leq l / 2}\left|e^{i \theta_{j}}-e^{i \theta_{k}}\right|^{2}\left|1-e^{i\left(\theta_{j}+\theta_{k}\right)}\right|^{2} \\
& \frac{1}{\pi^{(l-1) / 2} 2^{l-1}((l-1) / 2) !} \delta\left(\theta_{l}\right) \prod_{j=1}^{(l-1) / 2} \delta\left(\theta_{j}-\theta_{(l-1) / 2+j}\right)\left|1-e^{i \theta_{j}}\right|^{2} \\
& \times \prod_{1 \leq j<k \leq(l-1) / 2}\left|e^{i \theta_{j}}-e^{i \theta_{k}}\right|^{2}\left|1-e^{i\left(\theta_{j}+\theta_{k}\right)}\right|^{2}, \quad l \text { odd },
\end{aligned}
$$

and $\langle\cdot\rangle_{\mathrm{O}^{-}(l)}$ denotes an average with respect to the eigenvalue p.d.f. for random matrices from the classical group $\mathrm{O}^{-}(l)$,

$$
\begin{aligned}
& \frac{1}{\pi^{l / 2-1} 2^{l-2}(l / 2) !} \delta\left(\theta_{l-1}\right) \delta\left(\theta_{l}-\pi\right) \prod_{k=1}^{l / 2-1} \delta\left(\theta_{k}-\theta_{l / 2+k-1}\right)\left|1-e^{2 i \theta_{k}}\right|^{2} \\
& \times \prod_{1 \leq j<k \leq l / 2-1}\left|e^{i \theta_{j}}-e^{i \theta_{k}}\right|^{2}\left|1-e^{i\left(\theta_{j}+\theta_{k}\right)}\right|^{2}, \quad l \text { even } \\
& \frac{1}{\pi^{(l-1) / 2} 2^{l-1}((l-1) / 2) !} \delta\left(\theta_{l}-\pi\right) \prod_{j=1}^{(l-1) / 2} \delta\left(\theta_{j}-\theta_{(l-1) / 2+j}\right)\left|1+e^{i \theta_{j}}\right|^{2} \\
& \times \prod_{1 \leq j<k \leq(l-1) / 2}\left|e^{i \theta_{j}}-e^{i \theta_{k}}\right|^{2}\left|1-e^{i\left(\theta_{j}+\theta_{k}\right)}\right|^{2}, \quad l \text { odd. }
\end{aligned}
$$

Substituting (3.5) in (3.4) we see that

$$
\operatorname{Pr}\left(l_{n, m}^{\square} \leq l\right)=\frac{1}{s_{n, m}}\left[\alpha^{n-2 m}\right]\left\langle\prod_{k=1}^{l}\left(1+\alpha e^{i \theta_{k}}\right)\left(\sum_{j=1}^{l} e^{i \theta_{j}}\right)^{n}\right\rangle_{\mathrm{O}(l)},
$$

and substituting this in (3.2) then gives

$$
\operatorname{Pr}\left(l^{\square} \leq l\right)=e^{-\alpha z-z^{2} / 2}\left\langle\prod_{k=1}^{l}\left(1+\alpha e^{i \theta_{k}}\right) e^{z \sum_{j=1}^{l} e^{i \theta_{k}}}\right\rangle_{\mathrm{O}(l)}
$$

as first obtained by Baik and Rains [7].

As for the average (2.5), the average (3.11) in the case $\alpha=0$ also arises as a gap probability at the hard edge of a matrix ensemble. Thus consider the Laguerre symplectic ensemble, specified by the eigenvalue probability density function proportional to

$$
\prod_{l=1}^{N} \lambda_{l}^{a} e^{-\lambda_{l}} \prod_{1 \leq j<k \leq N}\left|\lambda_{k}-\lambda_{j}\right|^{4}, \quad \lambda_{l}>0
$$


For $a=2(n-N)+1, n \geq N$, this is realized by the distinct eigenvalues of matrices of the form $X^{\dagger} X$ with $X$ an $n \times N$ real quaternion Gaussian matrix (embedded as a complex matrix). Let $E_{4}^{\mathrm{L}}(s ; a ; N)$ denote the probability that there are no eigenvalues in the interval $(0, s)$. Then we know from [20, 11] that for $a \in \mathbb{Z}_{\geq 0}$

$$
E_{4}^{\mathrm{hard}}(\lambda ; 2 a):=\lim _{N \rightarrow \infty} E_{4}^{\mathrm{L}}\left(\frac{\lambda}{N} ; 2 a ; N / 2\right)=e^{-\lambda / 2}\left\langle e^{\sqrt{\lambda} \sum_{j=1}^{2 a} e^{i \theta_{j}}}\right\rangle_{\mathrm{O}(2 a)},
$$

thus coinciding with (3.11) in the case $\alpha=0$ and $z^{2}=\lambda, l=2 a$.

\subsection{The symmetry $\square$}

Analogous to the case of the symmetry $\square$, let us suppose points are added below the diagonal from $(0,1)$ to $(1,0)$ (to be referred to as the anti-diagonal) with rate $z d z$. Let the mirror images of these points in the anti-diagonal be also added, and let points on the anti-diagonal be added with rate $\beta d z$. Then we know from (3.1) and the following sentence that the probability there are exactly $n$ points after time $z, n-2 m$ of which are on the anti-diagonal, is equal to

$$
e^{-\beta z} e^{-z^{2} / 2} \frac{z^{n}}{n !} \beta^{n-2 m} s_{n, m}
$$

Now the permutation of $\{1,2, \ldots, n\}$ corresponding to a realization of this process is closely related to a realization of the symmetry $\square$. Thus let $P=(P(1), P(2), \ldots, P(n))$ be a permutation with the property that if $P(j)=k$ then $P(k)=j$ and thus correspond to the symmetry $\square$. Then $\tilde{P}:=(P(n), P(n-1), \ldots, P(1))$ has the property that if $\tilde{P}(j)=k$ then $\tilde{P}(n+1-k)=$ $n+1-j$ and so corresponds to the symmetry $\varangle$. Consequently the maximum length of the increasing subsequences in the case of $\nabla, l_{n, m}^{\triangleright}$ say, is equal to the maximum length of the decreasing subsequences for $\square$. Furthermore, it follows from a theorem of Greene [24] (see also [35]) relating row lengths of the standard tableau $\kappa$ corresponding to $P$, to increasing subsequences, and column lengths to decreasing subsequences, that the conjugate tableaux $\kappa^{\prime}$ obtained by interchanging the row and column lengths in $\kappa$, corresponds to $P^{R}$. Also, it follows from (3.3) that

$$
\text { \#(1's on the anti-diagonal })=\sum_{j=1}^{n}(-1)^{j-1} \kappa_{j}
$$

Setting $\kappa^{\prime}=\mu$, these facts together imply

$$
\begin{aligned}
\operatorname{Pr}\left(l_{n, m}^{\triangleright} \leq l\right) & =\frac{1}{s_{n, m}}\left[\beta^{n-2 m}\right] \sum_{\mu: \mu_{1} \leq l} \beta^{\sum_{j=1}^{n}(-1)^{j-1} \mu_{j}^{\prime}} f_{n}^{\mu} \\
& =\frac{1}{s_{n, m}}\left[\beta^{n-2 m} q_{1} \cdots q_{n}\right] \sum_{\mu: \mu_{1} \leq l} \beta^{\sum_{j=1}^{n}(-1)^{j-1} \mu_{j}^{\prime}} s_{\mu}\left(q_{1}, \ldots, q_{n}\right)
\end{aligned}
$$

(cf. (3.4)). In [7] it was shown that

$$
\sum_{\mu: \mu_{1} \leq 2 l} \beta^{\sum_{j=1}^{n}(-1)^{j-1} \mu_{j}^{\prime}} s_{\mu}\left(q_{1}, \ldots, q_{n}\right)=\left\langle\prod_{k=1}^{2 l}\left(\frac{1}{1-\beta e^{-i \theta_{k}}} \prod_{j=1}^{n}\left(1+q_{j} e^{i \theta_{k}}\right)\right)\right\rangle_{\operatorname{Sp}(l)}
$$


and

$$
\sum_{\mu: \mu_{1} \leq 2 l+1} \beta^{\sum_{j=1}^{n}(-1)^{j-1} \mu_{j}^{\prime}} s_{\mu}\left(q_{1}, \ldots, q_{n}\right)=\prod_{j=1}^{n}\left(1+\beta q_{j}\right)\left\langle\prod_{k=1}^{2 l} \prod_{j=1}^{n}\left(1+q_{j} e^{i \theta_{k}}\right)\right\rangle_{\operatorname{Sp}(l)}
$$

where \langle\rangle$_{\mathrm{Sp}(l)}$ denotes an average with respect to the eigenvalue p.d.f. for random matrices from the classical group $\operatorname{Sp}(l)$,

$$
\frac{1}{(2 \pi)^{l}} \frac{1}{2^{l} l !} \prod_{k=1}^{l} \delta\left(\theta_{k}-\theta_{l+k}\right)\left|e^{i \theta_{k}}-e^{-i \theta_{k}}\right|^{2} \prod_{1 \leq j<k \leq l}\left|e^{i \theta_{j}}-e^{i \theta_{k}}\right|^{2}\left|1-e^{i\left(\theta_{j}+\theta_{k}\right)}\right|^{2} .
$$

It follows immediately that

$$
\begin{aligned}
\operatorname{Pr}\left(l_{n, m}^{\triangleright} \leq 2 l\right) & =\frac{1}{s_{n, m}}\left[\beta^{n-2 m}\right]\left\langle\prod_{k=1}^{2 l} \frac{1}{1-\beta e^{-i \theta_{k}}}\left(\sum_{j=1}^{2 l} e^{i \theta_{j}}\right)^{n}\right\rangle_{\mathrm{Sp}(l)} \\
\operatorname{Pr}\left(l_{n, m}^{\triangleright} \leq 2 l+1\right) & =\frac{1}{s_{n, m}}\left[\beta^{n-2 m}\right]\left\langle\left(\beta+\sum_{j=1}^{2 l} e^{i \theta_{j}}\right)^{n}\right\rangle_{\mathrm{Sp}(l)} .
\end{aligned}
$$

Thus with

$$
\operatorname{Pr}\left(l^{\triangleright} \leq l\right):=e^{-\beta z-z^{2} / 2} \sum_{n=0}^{\infty} \frac{z^{n}}{n !} \sum_{m=0}^{n} \beta^{n-2 m} s_{n, m} \operatorname{Pr}\left(l_{n, m}^{\triangleright} \leq l\right)
$$

we have [7]

$$
\begin{aligned}
\operatorname{Pr}\left(l^{\triangleright} \leq 2 l\right) & =e^{-\beta z-z^{2} / 2}\left\langle e^{z \sum_{j=1}^{2 l} e^{i \theta_{j}}} \prod_{k=1}^{2 l} \frac{1}{1-\beta e^{-i \theta_{k}}}\right\rangle_{\mathrm{Sp}(l)} \\
\operatorname{Pr}\left(l^{\triangleright} \leq 2 l+1\right) & =e^{-z^{2} / 2}\left\langle e^{z \sum_{j=1}^{2 l} e^{i \theta_{j}}}\right\rangle_{\mathrm{Sp}(l)} \cdot
\end{aligned}
$$

There is an analogue of (3.12) for the second average in (3.16). For this consider the Laguerre orthogonal ensemble, specified by the eigenvalue probability density function proportional to

$$
\prod_{l=1}^{N} \lambda_{l}^{a} e^{-\lambda_{l} / 2} \prod_{1 \leq j<k \leq N}\left|\lambda_{k}-\lambda_{j}\right|, \quad \lambda_{l}>0
$$

For $a=(n-N-1) / 2, n \geq N$, this is realized by the distinct eigenvalues of matrices of the form $X^{T} X$ with $X$ an $n \times N$ real standard Gaussian matrix. Let $E_{1}^{\mathrm{L}}(s ; a ; N)$ denote the probability that there are no eigenvalues in the interval $(0, s)$. Then we know from [20, 11] that for $a \in \mathbb{Z}_{\geq 0}$

$$
E_{1}^{\mathrm{hard}}(\lambda ; a):=\lim _{N \rightarrow \infty} E_{1}^{\mathrm{L}}\left(\frac{\lambda}{N} ; a ; N\right)=e^{-\lambda / 2}\left\langle e^{\sqrt{\lambda} \sum_{j=1}^{2 a} e^{i \theta_{j}}}\right\rangle_{\mathrm{Sp}(a)},
$$

thus coinciding with (3.16) after setting $\lambda=z^{2}, a=l$.

\subsection{The symmetry $\otimes$}

For this symmetry only the points below both the diagonal and anti-diagonal are independent. Let us suppose points are added to this region with rate $2 z d z$. Suppose too that points are added 
to the bottom half of the diagonal with rate $\alpha d z$, and to the bottom half of the anti-diagonal with rate $\beta d z$. The images of all these points must also be added. The probability of there being exactly $2 n$ points after time $z, 2 m_{+}$of which are on the diagonal, and $2 m_{-}$of which are on the anti-diagonal, is then equal to

$$
\frac{z^{n}}{n !} e^{-z^{2}-\alpha z-\beta z} \alpha^{m_{+}} \beta^{m_{-}} t_{n, m_{+}, m_{-}}
$$

where

$$
t_{n, m_{+}, m_{-}}=\frac{n !}{m_{+} ! m_{-} !\left(\left(n-m_{+}-m_{-}\right) / 2\right) !}
$$

and it is required $n-m_{+}-m_{-}$be even.

The permutation matrix $P=\left[x_{i, j}\right]_{i, j=1, \ldots, 2 n}$ corresponding to a realization of this process has the two symmetries $P=P^{T}=\left[x_{j, i}\right]_{i, j=1, \ldots, 2 n}$ and $P=P^{R}=\left[x_{2 n+1-j, 2 n+1-i}\right]_{i, j=1, \ldots, 2 n}$. Now whereas a permutation matrix with the symmetry $P=P^{T}$ maps under the RSK correspondence to a pair of identical standard tableaux $\left(T_{1}, T_{1}\right)$ say, a permutation matrix with the symmetry $P=P^{R}$ maps to a pair of standard tableaux $\left(T_{2}^{R}, T_{2}\right)$ where $T_{2}^{R}$ denotes the Schützenberger dual of $T_{2}$ (see e.g. 35]). We note too that the number of permutation matrices of $\{1,2, \ldots, 2 n\}$ with the symmetry $P=P^{T}=P^{R}$ such that $m_{+}\left(m_{-}\right)$members of $\{1,2, \ldots, n\}$ have the property that $P(i)=i(P(i)=2 n+1-i)$ is equal to $t_{n, m_{+}, m_{-}}$. Hence with $l_{n, m_{+}, m_{-}}^{\bigotimes}$ denoting the longest path length in a realization of the Hammersley process with symmetry $\bowtie$, we have

$$
\operatorname{Pr}\left(l_{n, m_{+}, m_{-}}^{\bigotimes} \leq l\right)=\frac{\left[\alpha^{m_{+} \beta^{m_{-}}}\right]}{t_{n, m_{+}, m_{-}}} \sum_{\mu: \mu_{1} \leq l} \alpha^{\sum_{j=1}^{2 n}(-1)^{j-1} \mu_{j}} \beta^{\sum_{j=1}^{2 n}(-1)^{j-1}} \mu_{j}^{\prime} \tilde{f}_{2 n}^{\mu}
$$

where

$$
\tilde{f}_{2 n}^{\mu}=\#(\text { self dual standard tableaux, shape } \mu \text {, content } 2 n) .
$$

Analogous to (2.6), let us define

$$
\tilde{s}_{\lambda}^{\text {s.d. }}\left(q_{1}, \ldots, q_{n}\right)=\sum^{*} q_{1}^{\# 1^{\prime} s} \cdots q_{n}^{\# n^{\prime} s}
$$

where the asterisk denotes that the sum is over all self-dual semi-standard tableaux of shape $\lambda$ and content $2 n$. (For self dual tableaux of content $2 n$, \#j's $=\#(2 n+1-j)$ 's so there are $n$ rather than $2 n$ independent variables in the weightings.) From the definitions

$$
\tilde{f}_{2 n}^{\mu}=\left[q_{1} \cdots q_{n}\right] \tilde{s}_{\lambda}^{\text {s.d. }}\left(q_{1}, \ldots, q_{n}\right),
$$

so we can write

$$
\begin{gathered}
\operatorname{Pr}\left(l_{n, m_{+}, m_{-}}^{\bigotimes} \leq l\right)=\frac{1}{t_{n, m_{+}, m_{-}}}\left[\alpha^{m_{+}} \beta^{m_{-}} q_{1} \cdots q_{n}\right] \sum_{\mu: \mu_{1} \leq l} \alpha^{\sum_{j=1}^{2 n}(-1)^{j-1} \mu_{j}} \\
\quad \times \beta^{\sum_{j=1}^{2 n}(-1)^{j-1} \mu_{j}^{\prime}} \tilde{s}_{\lambda}^{\text {s.d. }}\left(q_{1}, \ldots, q_{n}\right) .
\end{gathered}
$$

Baik and Rains [7 have provided the evaluation

$$
\sum_{\mu: \mu_{1} \leq 2 l} \alpha^{\sum_{j=1}^{2 n}(-1)^{j-1} \mu_{j}} \beta^{\sum_{j=1}^{2 n}(-1)^{j-1} \mu_{j}^{\prime}} \tilde{s}_{\lambda}^{\text {s.d. }}\left(q_{1}, \ldots, q_{n}\right)=\left\langle\prod_{j=1}^{l} \frac{1+\alpha e^{i \theta_{j}}}{1-\beta e^{i \theta_{j}}} \prod_{k=1}^{n}\left|1+q_{k} e^{i \theta_{j}}\right|^{2}\right\rangle_{U(l)} .
$$


Hence

$$
\operatorname{Pr}\left(l_{n, m_{+}, m_{-}}^{\bowtie} \leq 2 l\right)=\left[\alpha^{m_{+}} \beta^{m_{-}}\right]\left\langle\prod_{j=1}^{l} \frac{1+\alpha e^{i \theta_{j}}}{1-\beta e^{i \theta_{j}}}\left(\sum_{k=1}^{l} 2 \cos \theta_{k}\right)^{n}\right\rangle_{U(l)}
$$

and consequently with

$$
\operatorname{Pr}\left(l^{\otimes} \leq 2 l\right):=e^{-z^{2}-\alpha z-\beta z} \sum_{n=0}^{\infty} \frac{z^{n}}{n !} \sum_{m_{+}, m_{-} \geq 0} \alpha^{m_{+}} \beta^{m_{-}} t_{n, m_{+}, m_{-}} \operatorname{Pr}\left(l_{n, m_{+}, m_{-}}^{\otimes} \leq 2 l\right)
$$

we obtain the result [7]

$$
\operatorname{Pr}\left(l^{\bowtie} \leq 2 l\right)=e^{-z^{2}-\alpha z-\beta z}\left\langle\prod_{j=1}^{l} \frac{1+\alpha e^{i \theta_{j}}}{1-\beta e^{i \theta_{j}}} e^{2 z \sum_{k=1}^{l} \cos \theta_{k}}\right\rangle_{U(l)} .
$$

Baik and Rains [7] have also provided the evaluation

$$
\begin{aligned}
& \sum_{\mu: \mu_{1} \leq 2 l+1} \alpha^{\sum_{j=1}^{2 n}(-1)^{j-1} \mu_{j}} \beta^{\sum_{j=1}^{2 n}(-1)^{j-1} \mu_{j}^{\prime}} \widetilde{s}_{\mu}^{\text {s.d. }}\left(q_{1}, \ldots, q_{n}\right) \\
& \quad=\prod_{k=1}^{n}\left(1+\beta q_{k}\right)\left\langle\prod_{j=1}^{l}\left(1+\alpha e^{i \theta_{j}}\right) \prod_{k=1}^{n}\left|1+q_{k} e^{i \theta_{j}}\right|^{2}\right\rangle_{U(l)} .
\end{aligned}
$$

We readily deduce from this that [7]

$$
\operatorname{Pr}\left(l^{\bowtie} \leq 2 l+1\right)=\left.\operatorname{Pr}\left(l^{\bowtie} \leq 2 l\right)\right|_{\beta=0} .
$$

\subsection{The symmetry $\odot$}

In the unit square suppose points are marked in the region $y<1 / 2$ with Poisson rate $4 z d z$. For each point $\left(x^{\prime}, y^{\prime}\right)$ so marked, also mark the image $\left(1-x^{\prime}, 1-y^{\prime}\right)$, which corresponds to a reflection about the centre of the square. A realization of this process with $2 n$ points corresponds to a permutation matrix with the symmetry $X=\left(X^{R}\right)^{T}$, or equivalently to a permutation of $\{1,2, \ldots, 2 n\}$ with the property that if $P(i)=j$, then $P(2 n+1-i)=2 n+1-j$. Note that there are $(2 n) ! !=2^{n} n$ ! permutations of this type.

Now we know [35] that if a permutation matrix $P$ maps to a pair of standard tableaux $\left(T_{1}, T_{2}\right)$ under the Robinson-Schensted mapping, then $P^{R}$ maps to $\left(T_{2}^{R}, T_{1}^{R}\right)$ while $P^{T}$ maps to $\left(T_{2}, T_{1}\right)$. Hence a permutation matrix with the symmetry $X=\left(X^{R}\right)^{T}$ maps to a pair of standard tableaux $\left(T_{1}, T_{2}\right)$ constrained so that

$$
T_{1}=T_{1}^{R}, \quad T_{2}=T_{2}^{R}
$$

Consequently with $l_{2 n}^{\square}$ denoting the longest path in a realization of $2 n$ points,

$$
\begin{aligned}
\operatorname{Pr}\left(l_{2 n}^{\square} \leq l\right) & =\frac{1}{2^{n} n !} \sum_{\mu: \mu_{1} \leq l}\left(\tilde{f}_{2 n}^{\mu}\right)^{2} \\
& =\frac{1}{2^{n} n !}\left[a_{1} \cdots a_{n} b_{1} \cdots b_{n}\right] \sum_{\mu: \mu_{1} \leq l} \tilde{s}_{\mu}\left(a_{1}, \ldots, a_{n}\right) \tilde{s}_{\mu}\left(b_{1}, \ldots, b_{n}\right) .
\end{aligned}
$$


Baik and Rains [7] have derived the results

$$
\begin{aligned}
& \sum_{\mu: \mu_{1} \leq 2 l} \tilde{s}_{\mu}\left(a_{1}, \ldots, a_{n}\right) \tilde{s}_{\mu}\left(b_{1}, \ldots, b_{n}\right) \\
& =\left(\left\langle\prod_{j=1}^{n} \prod_{k=1}^{l}\left(1+a_{j} e^{i \theta_{k}}\right)\left(1+b_{j} e^{-i \theta_{k}}\right)\right\rangle_{U(l)}\right)^{2}=\left\langle\prod_{j=1}^{n} \prod_{k=1}^{2 l}\left(1+a_{j} e^{i \theta_{k}}\right)\left(1+b_{j} e^{-i \theta_{k}}\right)\right\rangle_{U(l) \oplus U(l)} . \\
& \sum_{\mu: \mu_{1} \leq 2 l+1} \tilde{s}_{\mu}\left(a_{1}, \ldots, a_{n}\right) \tilde{s}_{\mu}\left(b_{1}, \ldots, b_{n}\right) \\
& \quad=\left\langle\prod_{j=1}^{n} \prod_{k=1}^{l+1}\left(1+a_{j} e^{i \theta_{k}}\right)\left(1+b_{j} e^{-i \theta_{k}}\right)\right\rangle_{U(l+1)}\left\langle\prod_{j=1}^{n} \prod_{k=1}^{l}\left(1+a_{j} e^{i \theta_{k}}\right)\left(1+b_{j} e^{-i \theta_{k}}\right)\right\rangle_{U(l)} \\
& \quad=\left\langle\prod_{j=1}^{n} \prod_{k=1}^{2 l+1}\left(1+a_{j} e^{i \theta_{k}}\right)\left(1+b_{j} e^{-i \theta_{k}}\right)\right\rangle_{U(l+1) \oplus U(l)}
\end{aligned}
$$

Consequently

$$
\begin{aligned}
\operatorname{Pr}\left(l_{2 n}^{\square} \leq 2 l\right) & =\frac{1}{2^{n} n !}\left\langle\left|\sum_{k=1}^{2 l} e^{i \theta_{k}}\right|^{2 n}\right\rangle_{U(l) \oplus U(l)} \\
\operatorname{Pr}\left(l_{2 n}^{\square} \leq 2 l+1\right) & =\frac{1}{2^{n} n !}\left\langle\left|\sum_{k=1}^{2 l+1} e^{i \theta_{k}}\right|^{2 n}\right\rangle_{U(l+1) \oplus U(l)}
\end{aligned}
$$

and thus with

$$
\operatorname{Pr}\left(l^{\square} \leq l\right):=e^{-2 z^{2}} \sum_{n=0}^{\infty} \frac{2^{n} z^{2 n}}{n !} \operatorname{Pr}\left(l_{2 n}^{\square} \leq l\right)
$$

we see that 7

$$
\begin{aligned}
& \operatorname{Pr}\left(l^{\square} \leq 2 l\right)=\left(\left.\operatorname{Pr}\left(l^{\square} \leq l\right)\right|_{\lambda \mapsto z}\right)^{2} \\
& \operatorname{Pr}\left(l^{\square} \leq 2 l\right)=\left.\left.\operatorname{Pr}\left(l^{\square} \leq l+1\right)\right|_{\lambda \mapsto z} \operatorname{Pr}\left(l^{\square} \leq l\right)\right|_{\lambda \mapsto z}
\end{aligned}
$$

where $\operatorname{Pr}\left(l^{\square} \leq l\right)$ is given by (2.5).

\section{The Hammersley process with sources on the boundary}

The Hammersley process which relates to the scaling function for one-dimensional stationary KPZ growth is the original model (Poisson points in a square), generalized to allow independent Poisson rates for points forming on the boundaries $y=0$ (with intensity $\alpha_{-}$) and $x=0$ (with intensity $\alpha_{+}$). In the PNG model picture, these boundary points correspond to growth from the boundary of the expanding droplet, with rate $\alpha_{+} d t$ on the left boundary, and rate $\alpha_{-} d t$ on the right boundary. Such boundary growths can be realized by an initial condition of mean slope $-\alpha_{-}\left(\alpha_{+}\right)$for $x<0(x>0)$, created by a staircase structure (downward sloping for $x<0$, upward sloping for $x>0$ ), with vertical increments of one unit with intensity $\alpha_{-}\left(\alpha_{+}\right)$. 
A formula for the cumulative probability of the longest increasing subsequence length of this model has been obtained by Baik and Rains [6]. This was obtained as a limiting case of an inhomogeneous lattice generalization of the Hammersley process due to Johansson [29].

In the Johansson model, on each site $(i, j), 0 \leq i, j \leq n$ of a $(n+1) \times(n+1)$ square grid there is a non-negative integer geometric random variable $x_{i, j}$ with parameter $a_{i} b_{j}$ so that

$$
\operatorname{Pr}\left(x_{i, j}=k\right)=\left(1-a_{i} b_{j}\right)\left(a_{i} b_{j}\right)^{k} .
$$

A type of directed last passage percolation is to form an $\mathrm{u} / \mathrm{rh}$ lattice path from the site $(0,0)$ to the site $(n, n)$ such that it maximizes the sum total of the random variables associated with the sites. Of interest then is the value of this maximized sum,

$$
L(n, n):=\max \sum_{(0,0) \mathrm{u} / \mathrm{rh}(n, n)} x_{i, j}
$$

It is not hard to show that in the RSK correspondence mapping the non-negative integer matrix $X=\left[x_{i, j}\right]_{i, j=0, \ldots, n}$ to a pair of semi-standard tableaux of the same shape and content $n+1$, the length of the first row of the tableaux is equal to $L(n, n)$ (one approach is to show that $L(n, n)$ and the height at the origin in the PNG model picture of the RSK correspondence satisfy the same recurrence). Further, one has that with entries of the matrix chosen according to (4.1), the RSK correspondence maps to pairs of weighted semi-standard tableaux, weights $\left\{a_{i}\right\},\left\{b_{j}\right\}$ repsectively. Consequently

$$
\operatorname{Pr}(L(n, n) \leq l)=\prod_{j, k=0}^{n}\left(1-a_{j} b_{k}\right) \sum_{\mu: \mu_{1} \leq l} s_{\mu}\left(a_{0}, \ldots, a_{n}\right) s_{\mu}\left(b_{0}, \ldots, b_{n}\right)
$$

and thus according to (2.8)

$$
\operatorname{Pr}(L(n, n) \leq l)=\prod_{j, k=0}^{n}\left(1-a_{j} b_{k}\right)\left\langle\prod_{j=0}^{n} \prod_{k=1}^{l}\left(1+a_{j} e^{i \theta_{k}}\right)\left(1+b_{j} e^{-i \theta_{k}}\right)\right\rangle_{U(l)}
$$

Suppose we set

$$
a_{i}=b_{i}=t / n \quad(i=1, \ldots, n)
$$

and take the limit $n \rightarrow \infty$. Then the probability that there are $k$ points in the region $1 \leq i, j \leq n$ has the large $n$ behaviour

$$
\left(\begin{array}{c}
n^{2} \\
k
\end{array}\right)\left(\left(1-\frac{t^{2}}{n^{2}}\right) \frac{t^{2}}{n^{2}}\right)^{k}\left(1-\frac{t^{2}}{n^{2}}\right)^{n^{2}-k} \rightarrow \frac{e^{-t^{2}} t^{2 k}}{k !}
$$

where the first factor corresponds to the number of different ways of choosing $k$ sites from the grid of $n^{2}$ sites, the second factor is the probability that those $k$ sites are occupied, while the final factor is the probability that the remaining sites are empty. Hence we reclaim the setting of the original Hammersley model with Poisson parameter $t^{2}$. Indeed with $a_{0}=b_{0}=0$ we see that by substituting (4.3) in (4.2) and taking the limit $n \rightarrow \infty$ (2.5) results with $\lambda=t$. 
Instead of setting $a_{0}$ and $b_{0}$ to zero, suppose we choose $a_{0}=\alpha_{+}, b_{0}=\alpha_{1}$. Then the argument leading to (4.4) shows that along $x>0(y>0)$ we obtain a Poisson process of intensity $\alpha_{+} t$ $\left(\alpha_{-} t\right)$. At the origin there remains a non-negative integer variable chosen according to the geometric distribution with parameter $\alpha_{+} \alpha_{-}, g\left(\alpha_{+} \alpha_{-}\right)$. Let us denote by $L^{+}\left(t, \alpha_{+}, \alpha_{-}\right)$the length of the longest up/right path in this process, and let us denote by $L\left(t, \alpha_{+}, \alpha_{-}\right)$the same quantity but with the geometric random variable at the origin removed. Clearly

$$
L^{+}\left(t, \alpha_{+}, \alpha_{-}\right)=L\left(t, \alpha_{+}, \alpha_{-}\right)+\chi, \quad \chi \in g\left(\alpha_{+} \alpha_{-}\right)
$$

while the appropriate limit of (4.2) gives

$$
\operatorname{Pr}\left(L^{+}\left(t, \alpha_{+}, \alpha_{-}\right) \leq l\right)=\left(1-\alpha_{+} \alpha_{-}\right) e^{-\left(\alpha_{+}+\alpha_{-}\right) t-t^{2}} \tilde{D}_{l}
$$

where

$$
\tilde{D}_{l}=\left\langle\prod_{j=1}^{l}\left(1+\alpha_{+} e^{i \theta_{j}}\right)\left(1+\alpha_{-} e^{-i \theta_{j}}\right) e^{2 t \sum_{j=1}^{l} \cos \theta_{j}}\right\rangle_{U(l)} .
$$

To now obtain a formula for $\operatorname{Pr}(L(t) \leq l)$, introduce the generating functions

$$
Q(x)=\sum_{l=0}^{\infty} \operatorname{Pr}(L(t) \leq l) x^{l}, \quad Q^{+}(x)=\sum_{l=0}^{\infty} \operatorname{Pr}\left(L^{+}(t) \leq l\right) x^{l} .
$$

Then using (4.5) we see that

$$
\begin{aligned}
Q^{+}(x) & =\sum_{l=0}^{\infty} x^{l} \sum_{k=0}^{l} \operatorname{Pr}(L(t) \leq l-k) \operatorname{Pr}(\chi=k) \\
& =\left(1-\alpha_{+} \alpha_{-}\right) \sum_{l=0}^{\infty} x^{l} \sum_{k=0}^{l} \operatorname{Pr}(L(t) \leq l-k)\left(\alpha_{+} \alpha_{-}\right)^{k}=\frac{1-\alpha_{+} \alpha_{-}}{1-x \alpha_{+} \alpha_{-}} Q(x)
\end{aligned}
$$

where the final equality follows by writing $x^{l}=x^{l-k} x^{k}$ and summing independently over $l-k$ and $k$. Multiplying both sides of this identity by $1-x \alpha_{+} \alpha_{-}$and equating like powers of $x$ gives [6]

$$
\operatorname{Pr}\left(L\left(t, \alpha_{+}, \alpha_{-}\right) \leq l\right)=e^{-\left(\alpha_{+}+\alpha_{-}\right) t-t^{2}}\left(\tilde{D}_{l}-\alpha_{+} \alpha_{-} \tilde{D}_{l-1}\right) .
$$

To proceed further, $\tilde{D}_{l}$ is expressed in terms of

$$
D_{l}=\left.\tilde{D}_{l}\right|_{\alpha_{+}=\alpha_{-}=0}=\left\langle e^{2 t \sum_{j=1}^{l} \cos \theta_{j}}\right\rangle_{U(l)},
$$

and monic orthogonal polynomials $\left\{\pi_{j}\left(e^{i \theta}\right)\right\}_{j=0,1, \ldots}$ with respect to the weight $e^{2 t \cos \theta}$,

$$
\frac{1}{2 \pi} \int_{-\pi}^{\pi} \pi_{j}\left(e^{i \theta}\right) \overline{\pi_{k}\left(e^{i \theta}\right)} e^{2 t \cos \theta} d \theta=\frac{1}{\kappa_{j}^{2}} \delta_{j, k} .
$$

For this purpose, let

$$
\pi_{n}^{*}(z):=z^{n} \pi_{n}\left(z^{-1}\right) .
$$


Then with $\left\{\pi_{j}\left(e^{i \theta}\right)\right\}_{j=0,1, \ldots}$ the monic orthogonal polynomials corresponding to the general real weight $w(\theta)$ replacing $e^{2 t \cos \theta}$ in (4.10), we have the general formula 36.

$$
\begin{aligned}
\frac{\left\langle\prod_{j=1}^{n} w\left(\theta_{j}\right)\left(e^{i \theta_{j}}-x\right)\left(e^{-i \theta_{j}}-y\right)\right\rangle_{U(n)}}{\left\langle\prod_{j=1}^{n} w\left(\theta_{j}\right)\right\rangle_{U(n)}} & =\frac{\pi_{n+1}^{*}(x) \pi_{n+1}^{*}(y)-\pi_{n+1}(x) \pi_{n+1}(y)}{1-x y} \\
& =\frac{\pi_{n}^{*}(x) \pi_{n}^{*}(y)-x y \pi_{n}(x) \pi_{n}(y)}{1-x y} \\
& =\frac{1}{\kappa_{n}^{2}} \sum_{k=0}^{n} \kappa_{k}^{2} \pi_{k}(x) \pi_{k}(y) .
\end{aligned}
$$

Application of the second of these equalities shows

$$
\tilde{D}_{l}=\frac{\pi_{l}^{*}\left(-\alpha_{+}\right) \pi_{l}^{*}\left(-\alpha_{-}\right)-\alpha_{+} \alpha_{-} \pi_{l}\left(-\alpha_{+}\right) \pi_{l}\left(-\alpha_{-}\right)}{1-\alpha_{+} \alpha_{-}} D_{l}
$$

and in particular, applying l'Hôpitals rule, we see that

$$
\left.\tilde{D}_{l}\right|_{\alpha_{+}=1 / \alpha_{-}=\alpha}=\left\{(1-l) \pi_{l}(-\alpha) \pi_{l}\left(-\alpha^{-1}\right)-\alpha \pi_{l}^{\prime}(-\alpha) \pi_{l}\left(-\alpha^{-1}\right)-\alpha^{-1} \pi_{l}(-\alpha) \pi_{l}^{\prime}\left(-\alpha^{-1}\right)\right\} D_{l} .
$$

The scaling behaviour of $D_{l}=D_{l}(t)$ as defined by (4.9) is the result (2.10) of Baik, Deift and Johansson. Thus we have

$$
\lim _{t \rightarrow \infty} e^{-t^{2}} D_{\left[2 t+t^{1 / 3} s\right]}(t)=F_{\mathrm{GUE}}(s) .
$$

We note that $F_{\mathrm{GUE}}(s)$ has the exact evaluation 37 ]

$$
F_{\mathrm{GUE}}(s)=\exp \left\{-\int_{s}^{\infty}(t-s) q^{2}(t) d t\right\}
$$

where $q(t)$ is the solution of the non-linear equation

$$
q^{\prime \prime}=t q+2 q^{3}
$$

(a special case of the Painlevé II equation) subject to the boundary condition

$$
q(t) \sim-\mathrm{Ai}(t) \quad \text { as } \quad t \rightarrow \infty
$$

where $\operatorname{Ai}(t)$ denotes the Airy function.

In addition to setting

$$
l=\left[2 t+t^{1 / 3} s\right]
$$

to obtain critical scaling behaviour, the parameter $\alpha$ must be related to $t$ by $[\underline{6}$

$$
\alpha=1-y / t^{1 / 3}
$$

where $y$ is fixed. To specify the corresponding scaled form of the orthogonal polynomials in (4.13), one establishes equations for their variation with respect to the scaled variables $s$ and $y$ 
6, 4, 33. In relation to the former, one recalls that in general monic orthogonal polynomials on the unit circle satisfy the coupled recurrences 36

$$
\begin{aligned}
& \pi_{n+1}(z)=z \pi_{n}(z)+r_{n+1} \pi_{n}^{*}(z) \\
& \pi_{n+1}^{*}(z)=r_{n+1} z \pi_{n}(z)+\pi_{n}^{*}(z)
\end{aligned}
$$

where $r_{n}=\pi_{n}(0)$. For the weight $w(z)=e^{2 t \cos \theta}$ the scaled form of $r_{l}=r_{l}(t)$ with the substitution (4.18) can be determined from a difference equation - a form of the discrete Painlevé II equation - satisfied by $\left\{r_{n}\right\}$ [32, 27, 39, 28, 4, 10, 1, 21].

Proposition 1. With $r_{n}:=\pi_{n}(0)$, the sequence $\left\{r_{n}\right\}$ for the polynomials with orthogonality (4.10) satisfies a form of the discrete Painlevé II equation

$$
-\frac{n}{t} \frac{r_{n}}{1-r_{n}^{2}}=r_{n+1}+r_{n-1}
$$

subject to the initial conditions

$$
r_{0}=1, \quad r_{1}=-\frac{I_{1}(t)}{I_{0}(t)}
$$

where $I_{\nu}(t)$ denotes the Bessel function of pure imaginary argument.

Proof. Following 28, let us show how (4.21) can be derived using simple properties of the weight

$$
w(z)=e^{t(z+1 / z)},
$$

together with a general formula from the theory of orthogonal polynomials on the unit circle. Regarding the latter, set $\phi_{j}(z):=\kappa_{j} \pi_{j}(z)$ so that according to (4.10) $\left\{\phi_{j}(z)\right\}$ defines an orthonormal set of polynomials on the unit circle, and introduce the coefficient $l_{n}$ by

$$
\phi_{n}(z)=\kappa_{n} z^{n}+l_{n} z^{n-1}+\cdots+\phi_{n}(0) .
$$

Then it is generally true that 36

$$
\frac{l_{n}}{\kappa_{n}}=\sum_{j=0}^{n-1} r_{j+1} \bar{r}_{j}, \quad r_{j}:=\frac{\phi_{j}(0)}{\kappa_{j}} .
$$

Specific to the weight (4.23) consider

$$
J:=\int_{\mathcal{C}} z^{2}\left(\frac{d}{d z} w(z)\right) \phi_{n}(z) \overline{\phi_{n+1}(z)} \frac{d z}{2 \pi i z},
$$

where $\mathcal{C}$ is a simple closed contour encircling the origin. Noting from (4.23) that

$$
\frac{d}{d z} w(z)=t\left(1-\frac{1}{z^{2}}\right) w(z)
$$

we see from integration by parts, the structure (4.24), and the orthonormality of $\left\{\phi_{j}(z)\right\}$ that

$$
J=-(n+1) \frac{\kappa_{n}}{\kappa_{n+1}}+(n+1) \frac{\kappa_{n+1}}{\kappa_{n}} .
$$


On the other hand, direct evaluation of $J$ using (4.24) and the orthonormality of $\left\{\phi_{j}(z)\right\}$ shows

$$
J=t\left(\frac{l_{n}}{\kappa_{n+1}}-\frac{l_{n+2}}{\kappa_{n+2}} \frac{\kappa_{n}}{\kappa_{n+1}}\right) .
$$

Equating (4.26) and (4.27) and eliminating $l_{n}$ using (4.25) (with $\overline{r_{j}}=r_{j}$ ) we arrive at (4.21).

Following [33], with

$$
R_{n}(t):=(-1)^{n-1} r_{n}(t)
$$

and making the ansatz

$$
R_{\left[2 t+t^{1 / 3} s\right]}(t) \sim t^{-1 / 3} u(s), \quad t \rightarrow \infty,
$$

we see that formally the difference equation (4.21) becomes the differential equation

$$
\frac{d^{2} u}{d s^{2}}=s u+2 u^{3} .
$$

Further, for (4.28) to be compatible with the first of the initial conditions (4.22), one must have

$$
u(s) \underset{s \rightarrow-\infty}{\sim}-\sqrt{-s / 2} .
$$

Now (4.29) is the same particular Painlevé II equation as (4.16). In fact it is a celebrated result [26] in the theory of the Painlevé II equation that (4.16) has a unique solution with the asymptotic $s \rightarrow-\infty$ behaviour (4.30), and the asymptotic $s \rightarrow \infty$ behaviour $u(s) \sim-\operatorname{Ai}(s)$ as in (4.17). Thus we conclude

$$
R_{\left[2 t+t^{1 / 3} s\right]}(t) \sim t^{-1 / 3} q(s), \quad t \rightarrow \infty .
$$

According to (4.13), our interest is in $\pi_{n}(-\alpha)$ and $\pi_{n}^{*}(-\alpha)$, so we should set $z=\alpha$ in (4.20). Introducing

$$
P_{n}(\alpha)=e^{-t \alpha} \pi_{n}^{*}(-\alpha), \quad Q_{n}(\alpha)=-e^{-t \alpha}(-1)^{n} \pi_{n}(-\alpha),
$$

one sees that (4.20) is consistent with the existence of the scaled quantities

$$
a(s, y):=\lim _{t \rightarrow \infty} P_{\left[2 t+t^{1 / 3} s\right]}\left(1-y / t^{1 / 3}\right), \quad b(s, y):=\lim _{t \rightarrow \infty} Q_{\left[2 t+t^{1 / 3} s\right]}\left(1-y / t^{1 / 3}\right),
$$

and that furthermore (4.20) reduces to the partial differential equations

$$
\frac{\partial a}{\partial s}=q b, \quad \frac{\partial b}{\partial s}=q a-y b,
$$

where use has also been made of (4.28) and (4.31). We note too that existence of the limits (4.33) together with the formula

$$
\left.\tilde{D}_{l}\right|_{\alpha_{+}=1 / \alpha_{-}=\alpha} / D_{l}=\frac{1}{\kappa_{l}^{2}} \sum_{k=0}^{l} \kappa_{k}^{2} \pi_{k}\left(\alpha_{+}\right) \pi_{k}\left(1 / \alpha_{-}\right),
$$

which follows from the final equality in (4.12), allows for the formal derivation of the limit

$$
\begin{gathered}
g(s, y):=\left.\lim _{t \rightarrow \infty} e^{-\left(\alpha_{+}+\alpha_{-}\right) t} \tilde{D}_{\left[2 t+t^{1 / 3} s\right]}(t)\right|_{\alpha_{+}=1 / \alpha_{-}=1-y / t^{1 / 3}} / D_{\left[2 t+t^{1 / 3} s\right]} \\
=\int_{-\infty}^{s} a\left(s^{\prime}, y\right) a\left(s^{\prime},-y\right) d s^{\prime}=\int_{-\infty}^{s} b\left(s^{\prime}, y\right) b\left(s^{\prime},-y\right) d s^{\prime}
\end{gathered}
$$


Similarly, at the same formal level, we see from (4.8), the definition of $g(s, y)$, and (4.14) that

$$
\begin{aligned}
\tilde{F}_{y}(s) & :=\lim _{t \rightarrow \infty} \operatorname{Pr}\left(\frac{L\left(t, 1-y / t^{1 / 3}, 1+y / t^{1 / 3}\right)-2 \sqrt{t}}{t^{1 / 6}} \leq s\right) \\
& =\frac{\partial}{\partial s}\left(g(s, y) F_{\mathrm{GUE}}(s)\right) .
\end{aligned}
$$

To fully determine $a$ and $b$, and thus $g(s, y)$ and the scaled distribution $\tilde{F}_{y}(s)$, it remains to specify equations for their dependence on the scaled variable $y$ as introduced in (4.19). Such equations follow from differential equations in $z$ for $\pi_{n}$ and $\pi_{n}^{*}$ [28, 4, 33.

Proposition 2. We have

$$
\begin{aligned}
\pi_{n}^{\prime}(z) & =\left(\frac{n}{z}+\frac{t}{z^{2}}-\frac{r_{n+1} r_{n} t}{z}\right) \pi_{n}(z)+\left(\frac{r_{n+1} t}{z}-\frac{r_{n} t}{z^{2}}\right) \pi_{n}^{*}(z) \\
\pi_{n}^{* \prime}(z) & =\left(-\frac{r_{n+1} t}{z}+r_{n} t\right) \pi_{n}(z)+\left(-t+\frac{r_{n+1} r_{n} t}{z}\right) \pi_{n}^{*}(z) .
\end{aligned}
$$

Proof. Let us show how (4.37) can be derived using results from [28]. In terms of the polynomials (4.10) for the weight (4.23), it is shown in [28, eq. (2.79) with $t \rightarrow 2 t$ ]

$$
\begin{aligned}
\phi_{n}^{\prime}(z)= & \frac{\kappa_{n-1}}{\kappa_{n}}\left(n+\frac{t}{z}+t \frac{\kappa_{n-1} \phi_{n-1}(0)}{\kappa_{n} \phi_{n}(0)}-t \frac{\phi_{n+1}(0) \phi_{n}(0)}{\kappa_{n+1} \kappa_{n}}\right) \phi_{n-1}(z) \\
& -\frac{t}{z} \frac{\kappa_{n-1}}{\kappa_{n}} \frac{\phi_{n-1}(0)}{\phi_{n}(0)} \phi_{n}(z) .
\end{aligned}
$$

But for a general weight 36

$$
\kappa_{n-1} z \phi_{n-1}(z)=\kappa_{n} \phi_{n}(z)-\phi_{n}(0) \phi_{n}^{*}(z), \quad 1-r_{n}^{2}=\left(\frac{\kappa_{n-1}}{\kappa_{n}}\right)^{2},
$$

which together with (4.24) show (4.39) reduces to (4.37).

The differential equations (4.37), (4.38) are consistent with the existence of the limits (4.33), and furthermore assume the scaled form

$$
\frac{\partial}{\partial y} a=q^{2} a-\left(q^{\prime}+y q\right) b, \quad \frac{\partial}{\partial y} b=\left(q^{\prime}-y q\right) a+\left(y^{2}-s-q^{2}\right) b .
$$

Together (4.34) and (4.40) determine the scaled quantities (4.33) once appropriate initial conditions are specified.

According to (4.19), when $y=0, \alpha=1$, so according to (4.33) we require the behaviour of $\pi_{n}(-1), \pi_{n}^{*}(-1)$. First we note from (4.11) that $\pi_{n}^{*}(-1)=(-1)^{n} \pi_{n}(-1)$ and thus (4.33) gives $a(s, 0)=-b(s, 0)$. It then follows from (4.34) that

$$
a(s, 0)=A e^{-U(s)}, \quad U(s)=-\int_{s}^{\infty} q(t) d t .
$$

To determine $A$, we note that formulas in [7] imply

$$
\lim _{n \rightarrow \infty}(-1)^{n} \pi_{n}(-1)=e^{t}
$$


so we see from (4.32) and (4.33) that

$$
a(s, 0) \rightarrow 1 \quad \text { as } \quad s \rightarrow \infty
$$

and so

$$
a(s, 0)=-b(s, 0)=e^{-U(s)} .
$$

The quantities $a(s, y), b(s, y)$ are now fully determined, and thus so to is $g(s, y)$ as specified by (4.35) and the scaled cumulative distribution $\tilde{F}_{y}(s)$ as specified by (4.36).

The scaled quantities satisfy a number of further properties of interest. First, with the initial condition (4.42) it is easy to see from (4.34) and (4.40) that $a(s, y)$ and $b(s, y)$ are related by

$$
a(s, y)=-b(s,-y) e^{\frac{1}{3} y^{3}-s y} .
$$

From this it is simple to verify that (4.35) can alternatively be written 7 ]

$$
g(s, y)=a(s,-y) \frac{\partial}{\partial y} a(s, y)-b(s,-y) \frac{\partial}{\partial y} b(s, y),
$$

which relates to (4.13). Second, it follows from (4.17), (4.34) and (4.42) that

$$
b(s, y) \underset{s \rightarrow \infty}{\sim}-e^{-y s} .
$$

One then sees from (4.43), (4.40) and (4.44) that

$$
g(s, y) \underset{s \rightarrow \infty}{\sim} s-y^{2} .
$$

Using this, the mean of the distribution $d \tilde{F}_{y} / d s$ can be computed as

$$
\int_{-\infty}^{\infty} s d \tilde{F}_{y}=\lim _{s \rightarrow \infty}\left(s \tilde{F}_{y}(s)-g(s, y) F_{\mathrm{GUE}}(s)\right)=y^{2}
$$

where the first equality follows from integration by parts and (4.36), while the second equality follows from the facts that $\tilde{F}_{y}(s)$ and $F_{\mathrm{GUE}}(s)$ approach 1 exponentially fast, together with (4.45). This then motivates defining the shifted cumulative distibution 33

$$
F_{y}(s):=\tilde{F}_{y}\left(s+y^{2}\right)
$$

for which the corresponding distribution function $d F_{y} / d s$ has mean zero. From the discussion in [33], ones sees that of immediate interest to KPZ growth is the second moment

$$
\int_{-\infty}^{\infty} s^{2} d F_{y}
$$

which is a function of the scaled parameter $y$. Prähofer and Spohn 33 have used the results revised above, evaluated using high precision computing, to accurately tabulate this quantity and discuss its properties. 


\section{$5 \quad$ Scaled limits for the symmetrized models}

In a remarkable analysis Baik and Rains [8] have provided similar evaluations to the one detailed in Section 4 of the scaled limits of the cumulative distributions in Section 3. Here we will be content with drawing attention to a subcase of two of these: $\operatorname{Pr}\left(l^{\square} \leq l\right)$ in the case $\alpha=0$ and $\operatorname{Pr}\left(l^{\square} \leq 2 l\right)$ in the case $\beta=0$. Thus it was first proved in [8] (see [1] for a subsequent simplified derivation based on the identities (3.12) and (3.17) that

$$
\begin{aligned}
& \lim _{z \rightarrow \infty} \operatorname{Pr}\left(\frac{l^{\square}(z)-2 z}{z^{1 / 3}} \leq s\right)=F_{\mathrm{GSE}}(s) \\
& \lim _{z \rightarrow \infty} \operatorname{Pr}\left(\frac{l^{\square}(z)-2 z}{z^{1 / 3}} \leq s\right)=F_{\mathrm{GOE}}(s) .
\end{aligned}
$$

Here $F_{\mathrm{GSE}}(s)$ denotes the scaled cumulative distribution of the largest eigenvalue for large Hermitian random matrices with real quaternion elements, while $F_{\mathrm{GOE}}(s)$ denotes the same for large real symmetric matrices.

Both $F_{\mathrm{GSE}}(s)$ and $F_{\mathrm{GOE}}(s)$ can be expressed in terms of the same Painlevé II transcendent as in the evaluation (4.15) of $F_{\mathrm{GUE}}(s)$. Thus one has 38.

$$
\begin{aligned}
& F_{\mathrm{GSE}}(s)=\frac{1}{2}\left(F_{\mathrm{GUE}}(s)\right)^{1 / 2}\left(e^{\frac{1}{2} \int_{s}^{\infty} q(t) d t}+e^{-\frac{1}{2} \int_{s}^{\infty} q(t) d t}\right) \\
& F_{\mathrm{GOE}}(s)=\left(F_{\mathrm{GUE}}(s)\right)^{1 / 2} e^{\frac{1}{2} \int_{s}^{\infty} q(t) d t} .
\end{aligned}
$$

Note the obvious inter-relationship

$$
F_{\mathrm{GSE}}(s)=\frac{1}{2}\left(F_{\mathrm{GOE}}(s)+\frac{F_{\mathrm{GUE}}(s)}{F_{\mathrm{GOE}}(s)}\right) .
$$

The origin of such an identity can be traced back to a special property of a particular marginal distribution of the joint probability for the row lengths of semi-standard tableaux relating to the symmetry $\varangle$ [7, 16, 18]. The marginal distribution is defined by summing over every second row

of the semi-standard tableaux. We also draw attention to the fact that $F_{\mathrm{GUE}}(s)$ and $F_{\mathrm{GOE}}(s)$ are $\tau$-functions for certain Painlevé II systems [37, 19]. Similarly, $F_{\mathrm{GSE}}(s)$ is the arithmetic mean of two $\tau$-functions, both of which correspond to Hamiltonians satisfying the same differential equation, differing only in the boundary condition [19].

\section{References}

[1] M. Adler and P. van Moerbeke. Recursion relations for unitary integrals, combinatorics and the Toeplitz lattice. math-ph/0201063, 2002.

[2] D. Aldous and P. Diaconis. Hammersley's interacting particle process and longest increasing subsequences. Prob. Theory and Rel. Fields, 103:199-213, 1995.

[3] D. Aldous and P. Diaconis. Longest increasing subsequences: from patience sorting to the Baik-Deift-Johansson theorem. Bull. Amer. Math. Soc., 36:413-432, 1999. 
[4] J. Baik. Riemann-Hilbert problem problems for last passage percolation. arXiv:math.PR/0107079.

[5] J. Baik, P. Deift, and K. Johansson. On the distribution of the length of the longest increasing subsequence of random permutations. J. Amer. Math. Soc., 12:1119-1178, 1999.

[6] J. Baik and E.M. Rains. Limiting distributions for a polynuclear growth model with external sources. J. Stat. Phys., 100:523-541, 2000.

[7] J. Baik and E.M. Rains. Algebraic aspects of increasing subsequences. Duke Math. J., 109:1-65, 2001.

[8] J. Baik and E.M. Rains. The asymptotics of monotone subsequences of involutions. Duke Math. J., 109:205-281, 2001.

[9] A.L. Barabási and H.E. Stanley. Fractal concepts in surface growth. Cambridge University Press, 1995.

[10] A. Borodin. Discrete gap probabilities and discrete Painlevé equations. Duke Math. J., 117:489-542, 2003.

[11] A. Borodin and P.J. Forrester. Increasing subsequences and the hard-to-soft transition in matrix ensembles. J.Phys. A, 36:2963-2981, 2003.

[12] M.E. Fisher. Walks, walls, wetting and melting. J. Stat. Phys., 34:667-729, 1984.

[13] P.J. Forrester. Exact solution of the lockstep model of vicious walkers. J. Phys. A, 23:1259$1273,1990$.

[14] P.J. Forrester. Exact results and universal asymptotics in the Laguerre random matrix ensemble. J. Math. Phys., 35:2539-2551, 1993.

[15] P.J. Forrester. Random walks and random permutations. J. Phys. A, 34:L417-L423, 2001.

[16] P.J. Forrester and E.M. Rains. Inter-relationships between orthogonal, unitary and symplectic matrix ensembles. In P.M. Bleher and A.R. Its, editors, Random matrix models and their applications, volume 40 of Mathematical Sciences Research Institute Publications, pages 171-208. Cambridge University Press, United Kingdom, 2001.

[17] P.J. Forrester and E.M. Rains. Correlations for superpositions and decimations of Laguerre and Jacobi orthogonal matrix ensembles with a parameter. math-ph/0211041

[18] P.J. Forrester and E.M. Rains. Interpretations of some parameter dependent generalizations of classical matrix ensembles. math-ph/0211042

[19] P.J. Forrester and N.S. Witte. $\tau$-function evaluations of gap probabilities in orthogonal and symplectic matrix ensembles. Nonlinearity 15: 937-954, 2001. 
[20] P.J. Forrester and N.S. Witte. Application of the $\tau$-function theory of Painlevé equations to random matrices: PVI, the JUE,CyUE, cJUE and scaled limits. math-ph/0204008, 2002.

[21] P.J. Forrester and N.S. Witte. Discrete Painlevé equations and random matrix averages. math-ph/0305029, 2003.

[22] W. Fulton. Young Tableaux. London Mathematical Society Student Texts. CUP, Cambridge, 1997.

[23] I.M. Gessel. Symmetric functions and p-recursiveness. J. Comb. Th. A, 53:257-285, 1990.

[24] C. Greene. An extension of Schensted's theorem. Adv. in Math., 14:254-265, 1974.

[25] T. Halpin-Healy and Y.C. Zhang. Kinetic roughening phenomena, stochastic growth, directed polymers and all that. Phys. Rep., 254:215-415, 1995.

[26] S.P. Hastings and J.B. McLeod. A boundary value problem associated with the second Painlevé transcendent and the Korteweg-de Vries equation. Arch. Rat Mech. Anal., 73:3151, 1980.

[27] M. Hisakado. Unitary-matrix models and Painlevé III. Mod. Phys. Lett. A, 11:3001-3010, 1996.

[28] M.E.H. Ismail and N.S. Witte. Discriminants and functional equations for polynomials orthogonal on the unit circle. J. Approx. Theory, 110:200-228, 2001.

[29] K. Johansson. Shape fluctuations and random matrices. Commun. Math. Phys., 209:437476, 2000.

[30] K. Johansson. Non-intersecting paths, random tilings and random matrices. Prob. Theory Rel. Fields, 123:225-280, 2002.

[31] D.E. Knuth. Permutations, matrices and generalized Young tableaux. Pacific J. Math., 34:709-727, 1970.

[32] V. Periwal and D. Shevitz. Unitary-matrix models as exactly solvable string theories. Phys. Rev. Lett., 64:1326-1329, 1990.

[33] M. Prähofer and H. Spohn. Exact scaling functions for one-dimensional stationary KPZ growth. arXiv:cond-mat/0212519, 2002.

[34] E.M. Rains. Increasing subsequences and the classical groups. Elect. J. of Combinatorics, 5:\#R12, 1998.

[35] B.E. Sagan. The symmetric group. Springer-Verlag, New York, 2nd edition, 2000.

[36] G. Szegö. Orthogonal polynomials. American Mathematical Society, Providence R.I., 4th edition, 1975. 
[37] C.A. Tracy and H. Widom. Level-spacing distributions and the Airy kernel. Commun. Math. Phys., 159:151-174, 1994.

[38] C.A. Tracy and H. Widom. On orthogonal and symplectic matrix ensembles. Commun. Math. Phys., 177:727-754, 1996.

[39] C.A. Tracy and H. Widom. Random unitary matrices, permutations and Painlevé. Commun. Math. Phys., 207:665-685, 1999.

[40] G. Viennot. Une forme géométrique de la correspondance de Robinson-Schensted. Lecture notes in Math., 579:29-58, 1977. 